\title{
Nonlinear three-dimensional flows in magnetised plasmas
}

\author{
K. Hallatschek \\ Max-Planck-Institut für Plasmaphysik, EURATOM Association, D-85748 \\ Garching, Germany \\ E-mail: hallatschek@ipp.mpg.de
}

\begin{abstract}
After a long history of theoretical predictions, turbulence induced poloidal flows - "Zonal Flows" (ZF) - are nowadays ubiquitously detected in tokamaks and stellarators. The difference in character of ZFs in a torus in comparison to those in a cylinder is discussed. The reduction in symmetry leads to a fundamentally three-dimensional flow pattern, a second oscillating flow type, and several additional interaction mechanisms between flows and turbulence equal in importance to the perpendicular Reynolds stress of the two-dimensional flows in a cylinder.
\end{abstract}

PACS numbers: 52.35.Ra, 52.55.Fa, 52.65.Kj

Submitted to: Plasma Phys. Control. Fusion

\section{Introduction}

Since the first identification of global oscillating poloidal flow activity in the D3D tokamak [1] - so called geodesic acoustic modes [2] (GAM) - these and even the harder to measure stationary "Zonal Flows" (ZF) have been observed in many more magnetic confinement devices (AUG, CHS, H-1, HL-2A, JFT-2M, Jet, JIPP-T2, Textor, TJ2 , T-10) $[3,4,5,6,7,8,9,10,11,12]$ and have turned out to be an ubiquitous phenomenon. As one of the rare successes of plasma theory, both types of flow had been predicted beforehand by analytic considerations $[13,14]$ and numerical turbulence simulations $[15,16,17]$. In contrast to the majority of plasma excitations, the flows are not directly driven by gradients in temperature and density but are secondary instabilities of the gradient driven turbulence $[18,19]$ or neoclassical heat flows (via variants of the Stringer instability [20,21]). For this reason, they are beneficial to confinement and a hot topic in controlled fusion research.

\section{Zonal flow observations}

Zonal flows were originally known just as planet-spanning latitudinal winds. Particularly striking are the ZFs on Jupiter, which are connected to the brown and white stripes [22]. Successful models for the generation of Jovian ZFs are based on the special properties of two-dimensional turbulence. The atmospheric turbulence is 
hypothesised to be quasi-two-dimensional either due to vortex alignment by the fast rotation [23], or due to the restriction of the turbulence to a thin weather layer [24].

The recognition that a strong magnetic field aligns plasma convective roles in an analogous way to the planetary Coriolis forces led to the prediction of plasma zonal flows based on inverse cascades as early as 1979 [13]. Probably the first computer simulations of ZFs in a magnetised plasma were those of Hasegawa and Wakatani [15]. The computations treated three-dimensional drift-wave turbulence in a cylindrical plasma with sheared magnetic field for cold ions and isothermal electrons. In the absence of particle sources or sinks, an initial burst of turbulence produces a poloidal $\boldsymbol{E} \times \boldsymbol{B}$-flow, which is strong enough to completely quench the turbulence and prevent further decay of the density gradient. Here, potential vorticity $\psi \equiv \nabla_{\perp}^{2}(\tilde{n}+\tilde{\phi})$ is exactly conserved, whence the system evolves into a state of minimum free energy density $f \equiv \tilde{n}^{2} / 2+\left(\nabla_{\perp} \tilde{\phi}\right)^{2} / 2[25]$ at given mean square potential vorticity, which is characterised by a finite gradient and stable poloidal flows. In contrast, the flows parallel to the field have been shown to be unstable against a specific drift-instability [26] and are not observed to play a significant role in the turbulence simulations.

The behaviour of the Hasegawa-Wakatani-system can be regarded as the limiting case of the general situation with less conserved quantities. Tokamak core simulations with realistic toroidal geometry within the gyrofluid [16] and gyrokinetic framework $[17,27]$ also found turbulence generated ZFs. These flows usually just reduce the turbulence level by a factor 4-10. Complete suppression occurs only very close to marginal stability seemingly upshifting the instability threshold [28]. The flows' tendency to reduce the turbulence is caused by the shearing of the turbulence [29] and by drawing on the turbulence free energy (kinetic energy and fluctuation energy by modification of the cross phase of fluctuations in potential and particle distribution). While the latter effect is the dominant one in the cylindrical drift wave scenario, the former is dominant for toroidal instabilities - these are trapped at the outboard midplane and cannot easily "unshear" themselves by propagating along the field lines. (The turbulence reduction effect and the observed $\boldsymbol{E} \times \boldsymbol{B}$-flow feature connected with the H-mode was the dominant motivation for initial analytic studies of flows $[29,14,30]$.

From the core studies, the flows in a torus appeared to be rather similar to the cylindrical ones. In contrast, toroidal edge turbulence computations appear not to have yielded significant stationary flows. In retrospect, the flows observed in some of them (in pursuit of the H-mode [31]) seem to be either transient due to parameter changes and initialisation effects, or due to GAMs. On the other hand, GAMs, oscillating flow modes [2] (some more details follow), are frequently encountered in edge simulations [32] while being of minor importance for the core turbulence case [33].

Facilitated by the spectral peak caused by their finite oscillation frequency, these GAMs have been identified in tokamaks well before the stationary zonal flows [1] by Fourier analysing the Doppler spectroscopy signal of the turbulence. The stationary ZFs have been identified much later by double heavy ion beam probes in CHS [4]. Nowadays it seems to be experimentally settled that GAMs and stationary ZFs occur ubiquitously in the edge and the core of tokamak plasmas, respectively $[3,4,5,6,7,8,9,10,11,12]$. 


\section{3D-Effect: toroidicity, GAMs and residual flows}

The superficial similarity of the ZFs in toroidal core turbulence and cylindrical drift wave simulations suggests that the mechanism for generation, control of the amplitude, and turbulence interaction are identical. So for simplicity most analytic studies have been carried out in cylinder or slab geometry [14, 18, 19, 29, 30], even if the turbulent modes have been described toroidally [34]. Unfortunately, this simplification disregards that the ZFs completely loose the character of a steady poloidal flow in toroidal geometry. The ZF-Eigenmodes split into a stationary and an oscillating branch. The stationary flows are thereby more properly regarded as parallel (or toroidal) flows with a rather subdominant poloidal component coupled to them. The consequences of this character change have been highlighted by recent turbulence computer studies [35, 36].

The toroidal effect on the ZFs can be deduced most clearly from the frozen magnetic flux condition in a low- $\beta$ plasma. On one hand the magnetic flux is frozen into the plasma, and thus swept with the flow. Thereby the flux density changes according to the divergence of the plasma flow perpendicular to the magnetic field. Since on the other hand the magnetic field can be assumed to be time-constant due to low $\beta$, the perpendicular flow divergence must be such that it continuously adjusts the frozen-in magnetic flux density to the ambient flux density. Since the magnetic field varies inversely proportional to the distance from the curvature centre, $B \propto 1 / R$, the perpendicular cross-section of a flux tube swept with the flow must change proportional to $R$. In addition the arc-length of the field lines varies $\propto R$, whence the volume of the plasma trapped in the magnetic field must be proportional $R^{2}$ and its density proportional to $1 / R^{2} \propto B^{2}$.

Taking as an example the ASDEX Upgrade tokamak, a fluid element moving from the outboard midplane at $R_{o}=2.1 \mathrm{~m}$ to the inboard midplane at $R_{i}=1.1 \mathrm{~m}$ is compressed by a factor $n_{i} / n_{o}=R_{o}^{2} / R_{i}^{2}=3.6$. The pressure - and the thermal energy density - would change adiabatically by a factor $p_{i} / p_{o}=\left(n_{i} / n_{o}\right)^{5 / 3}=8.6$. It is certain that the small residual flux surface averaged turbulence forces responsible for the ZFs are incapable of causing such an enormous pressure increase, exceeding by far the internal energy density. Instead, any poloidal momentum will just cause a small motion, which is immediately stopped and reversed by strong restoring forces due to the plasma compression. What results is an oscillation, the "geodesic acoustic mode" (as the compression is caused by the geodesic curvature-component of the field lines, i.e., the component tangential to the flux surface).

From this consideration - which is just a direct application of the cylindrical ZF concept to the toroidal case - it seems logical to conclude, that turbulence induced stationary flows are completely ruled out in toroidal systems, with the possible exception of (unreasonably) slender high aspect ratio machines. However, an altered form of the stationary ZFs is sometimes saved by another effect absent in a cylinder: the generation of a flow component parallel to the field lines, which cancels the plasma compression due to field curvature. Since the parallel component is susceptible to instabilities and turbulent damping, the toroidal ZFs are clearly weaker than the ones in a cylinder. The crucial question is now whether they retain the character of a poloidal flow or essentially turn into a parallel flow pattern.

Taking for example a circular high aspect ratio tokamak, the poloidal flow 
divergence due to the frozen flux argument is

$$
\nabla \cdot \boldsymbol{v}_{\mathrm{p}}=\boldsymbol{v}_{\mathrm{p}} \cdot \nabla \ln R^{2}=2 \frac{v_{\mathrm{p}, R}}{R}=2 \frac{v_{\mathrm{p}} \sin \theta}{R},
$$

where $v_{\mathrm{p}}$ is the poloidal velocity and $v_{\mathrm{p}, R}$ is its (major) radial component. This has to be cancelled by the parallel flow divergence

$$
\nabla \cdot \boldsymbol{v}_{\|}=\partial_{\|} v_{\|}=\frac{B_{\theta}}{B r} \partial_{\theta} v_{\|} \approx \frac{B_{\theta}}{B_{\phi} r} \partial_{\theta} v_{\|}=\frac{1}{q R} \partial_{\theta} v_{\|}
$$

where $q$ is the safety factor, the number of toroidal windings per poloidal winding of a field line. This balance is solved by $v_{\|}=-2 q v_{\mathrm{p}} \cos \theta$. The parallel kinetic energy density averaged over $\theta$ (indicated by \langle\rangle ) is

$$
\frac{\rho}{2}\left\langle v_{\|}^{2}\right\rangle=\frac{\rho}{2}\left\langle 4 q^{2} v_{\mathrm{p}}^{2} \cos ^{2} \theta\right\rangle=2 q^{2} \frac{\rho}{2} v_{\mathrm{p}}^{2}
$$

where $\rho$ is the mass density. The parallel kinetic energy can be described by an effective poloidal mass density as

$$
\frac{\rho}{2}\left\langle v_{\|}^{2}+v_{\mathrm{p}}^{2}\right\rangle=\frac{\rho}{2}\left(1+2 q^{2}\right)\left\langle v_{\mathrm{p}}^{2}\right\rangle \equiv \frac{\rho_{\text {eff. }}}{2}\left\langle v_{\mathrm{p}}^{2}\right\rangle
$$

Since in a tokamak $q$ varies from 1 in the core to $3-5$ near the edge, the parallelflow energy is at least a factor of 2 larger than the poloidal-flow energy. Therefore a stationary $\mathrm{ZF}$ is always more of a parallel flow than a poloidal one. Approaching the edge the ratio becomes $18-50$ and the poloidal flow component all but disappears! In this light, the absence of stationary poloidal flows in edge turbulence simulations appears quite natural.

For collisionless plasmas, the enhancement of the effective mass (identical to the inverse fraction of residual flow or the neoclassical enhancement of polarisation) has been computed by Rosenbluth and Hinton $[37,38]$ as $\rho=\rho_{\text {effective }}\left(1+1.6 q^{2} \sqrt{R / a}\right)$, provided $R \gg a$. The collisionless inertia is always larger than the fluid one, since all the divergences of the poloidal heat flux (and even higher moment fluxes) have to be cancelled by separate parallel flows, increasing the parallel kinetic energy. For wavelengths below the banana width, the polarisation suffers cut-off effects, which results in a contribution proportional to $\lambda_{r}^{2}\left(\lambda_{r}\right.$ is the $\mathrm{ZF}$ radial wavelength). The same always happens in a stellarator $[39,40]$, whose unconfined orbits can be regarded as infinitely wide banana orbits.

The GAM frequency can be derived by balancing the time averages of compressional and kinetic energy. The specific energy invested in compressing a fluid element of volume $V$, so that the pressure increases by $\delta p$, is

$$
\frac{\delta E_{\mathrm{c}}}{V}=-\frac{\delta p \delta V}{2 V}=\frac{\gamma p}{2}\left(\frac{\delta n}{n}\right)^{2}=\frac{\gamma p}{2}\left(2 \frac{\xi_{\mathrm{p}} \sin \theta}{R}\right)^{2},
$$

where $\delta V$ is the volume change, the factor $1 / 2$ arises because during compression the resistance rises from zero to the full $\delta p$, and $\gamma$ stems from the adiabatic gas law $p n^{-\gamma}=$ const. The relative change in density was obtained from the time integral of (1). With $\left\langle\sin ^{2} \theta\right\rangle=1 / 2$ and the reference sound speed $c_{s}=\sqrt{p / \rho}=\sqrt{\left(T_{i}+T_{e}\right) / m_{i}}$, the time and flux surface averaged energy balance reads

$$
\rho \frac{\overline{v_{\mathrm{p}}^{2}}}{2}=\rho \frac{\omega^{2} \overline{\xi_{\mathrm{p}}^{2}}}{2} \equiv \overline{\left\langle\frac{\delta E_{\mathrm{c}}}{V}\right\rangle}=\gamma p \frac{\overline{\xi_{\mathrm{p}}^{2}}}{R^{2}} \Rightarrow \omega=\sqrt{\frac{2 \gamma p}{\rho R^{2}}}=\frac{\sqrt{2 \gamma} c_{s}}{R} .
$$

In a tokamak plasma it is usually best to regard the electrons as isothermal $\left(\gamma_{e}=1\right)$ and the ions as adiabatic $\left(\gamma_{i}=5 / 3\right)$, whence the coefficient to be used is the mean, 
$\gamma=4 / 3$. The collisionless correction is $\omega_{\text {coll.less }}=\sqrt{33 / 32} \omega_{\text {fluid }}[41]$. (This can also be obtained by computing the effective $\gamma_{i, \text { kinetic }}=7 / 4 \Rightarrow \gamma_{\text {kinetic }}=11 / 8=33 / 32 \gamma_{\text {fluid }}$.

Judging from the frozen flux argument, GAM oscillation should exist quite generally in curved magnetic fields which at least partially follow flux surfaces. (For $\beta \sim 1$, the magnetic field is not rigid and contributes to the energy balance.) An astrophysical example may have been found by SUMER (solar UV spectrometer) on the SOHO space craft [42]: Observed were slow Doppler oscillations of solar coronal loops with periods of order of $10 \mathrm{~min}$, which are typical sound wave frequencies, albeit the characteristic intensity oscillations were absent in the observations. Interpreted as GAMs - with equally compatible frequency -, no intensity oscillations are expected, since the sight line averages over the expanding and compressing side of the coronal loop.

\section{Zonal flow generation and damping}

While ultimately the quasi two-dimensional character of the turbulence is responsible for the spin up of the otherwise stable ZFs, the precise reasons depend on the specific scenario. Generic hydrodynamic 2D-turbulence transfers energy in small spectral steps from small scales up to the largest scale flows, i.e., in an inverse energy cascade [43]. (This counter-intuitive flow of energy is required by the conservation of enstrophy in 2D.) Somewhat differently, in magnetised plasmas the turbulence (ion-temperaturegradient modes, resistive ballooning modes, drift waves, trapped electron modes, etc.) is confined to well defined wavenumber bands at scales much smaller than the minor radius. (If an instability actually does reach up to the largest scales, such as ideal ballooning modes, the result is usually catastrophic.) The energy is transferred in one big nonlocal spectral step from the turbulence to the flows. Since a large scale flow covers many turbulence eddies, the average force on the flow is well-defined and can be represented by the divergence of the turbulent transport of poloidal momentum, the Reynolds stress, which depends deterministically on the particular circumstances (not the least of which is the presence of ZFs themselves). The majority of analytic ZF studies have been devoted to the various mechanisms which can induce the turbulence to produce poloidal Reynolds stress.

One mechanism depends on the dispersive nature and non-zero group velocity of most of the turbulence modes. The Reynolds stress is often roughly proportional to the radial group velocity, or synonymously, the Poynting vector [14] of the modes. In case of a strong spatial variation of the turbulence level, the turbulence modes tend to drift from the more turbulent region to the stable region, while at the same time transferring poloidal momentum and creating a poloidal flow. This idea was later refined towards the "beach model" of the H-mode [30]. Moreover, consider a mode with vanishing $v_{\text {group }, r} \equiv \partial_{k_{r}} \omega=0$ at particular radial and poloidal wavenumber, $k_{r}$ and $k_{\theta}$, respectively. An ambient shear flow will alter the radial wavenumber with time, $k_{r}(t)=-t k_{\theta} \partial_{r} v_{\theta}+k_{r}(0)$, which subsequently causes non-zero radial group velocity. Due to their influence on the group velocity, ZFs can accelerate, brake or even trap wave packets. Since the group velocity entails Reynolds stress, wavetrapping amplifies the flow if the signs in the dispersion relation are right. This type of argument makes the theory completely analogous to the theory of kinetic plasma instabilities, opening up a rich field of applications and extensions. Examples are the modulational instability [18, 19], its extension to toroidal waves [34], theory of the nonlinear evolution and saturation [44] (loosely speaking if the wave-troughs are full), 


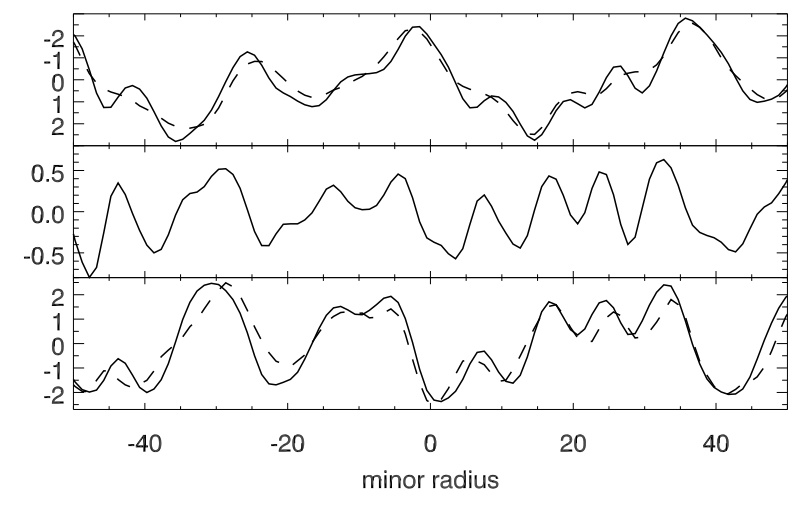

Figure 1. Instantaneous profiles at a later stage in an ITG simulation from [35]. Top: poloidal flow $\left\langle v_{\theta}\right\rangle$ (solid), parallel return flow $-\left\langle\cos \theta v_{\|}\right\rangle / q$ (dashed). Middle: shearing rate $\left\langle\partial_{r} v_{\theta}\right\rangle$. Bottom: poloidal Reynolds stress, negative effective parallel stress $2 q\left\langle\tilde{v_{r}} \tilde{v_{\|}} \cos \theta\right\rangle$. Note the good match between flow and return flow, poloidal and parallel stress, and the correlation between shearing rate and stress.

and application of the theory to large scale convective cells [45].

The results of these theories should not be taken too literal, since in reality tokamak turbulence cannot be considered weak. Nevertheless, dispersive wave trapping and the proportionality between Reynolds stress and Poynting vector can be readily observed in the simulations (see e.g. in [32]). Alas, the wave troughs are rather "leaky" for toroidal modes and the strong nonlinear interactions prevent the modes from travelling significant distances.

In the case of very strong turbulence or non-dispersive modes, far less can be said. Still, for sufficiently anisotropic turbulence, a negative turbulence viscosity, and hence an amplification of ambient flows is predicted [46].

All of the above theories regard the large scale ZFs as purely poloidal flows (even if the enhanced inertia is taken into account) and should strictly speaking be applied only to a cylinder. As we have seen in section 3, the parallel flow component is necessary for the flow to persist. Without it, plasma compression occurs, resulting in a restoring force stopping the flow.

In fact, the linear braking of weakly collisional ZFs by collisions can be viewed as the damping of the parallel flow component by collisions with a damping rate of order $\nu_{i i}[38]$. If only the perpendicular component were affected by collisions, the damping rate would be smaller by the effective inertia factor $\sim\left(1+1.6 q^{2} / \sqrt{\epsilon}\right)^{-1}$.

Much more important, the turbulence tends to reshuffle the parallel momentum in radial direction. Detailed data about the radial transport of the parallel ZF-component can be gleaned up to now only from turbulence simulations. It turns out that the flows evolve into an equilibrium between the driving poloidal Reynolds-stress and the turbulent braking force on the parallel flow component [35, 36] as shown in figure 1 . Were it not for the parallel braking, the ZFs would grow rapidly (within one sound transit time) and to much higher amplitudes. (It is thus inappropriate to search for the control mechanism for the flows by only looking at the poloidal Reynolds stress.)

To judge the importance of the parallel momentum diffusion, one may apply the mixing length estimate to both the poloidal negative turbulence viscosity and to the parallel positive turbulence viscosity. The turbulent momentum diffusion 
coefficient should thus be of the same order as the anomalous heat diffusion coefficient. (This is supported by the experimental finding that toroidal momentum and energy diffuse equally fast [47], noting that there is little difference between parallel and toroidal momentum in a tokamak.) Both viscosities lead therefore to the same kinetic energy amplification/damping rate, respectively. The difference is that the poloidal viscosity acts only on the poloidal flow, whose energy density is a factor $1 /\left(2 q^{2}\right)$ $\left(\sqrt{\epsilon} /\left(1.6 q^{2}\right)\right.$ collisionless) smaller than the parallel flow's energy density. Thus the damping increases relative to the drive proportional to $q^{2}$. This is the reason for the complete absence of stationary ZFs in the edge region of tokamaks, where $q \sim 3-5$. (On a side note, this fact confounds H-mode theories based on the amplification of poloidal flows by Reynolds stress.) It may be mentioned that the parallel flows are also subject to a drift type instability [26]. For non-marginal ITG turbulence this instability seems, however, to be negligible in comparison to the turbulent damping [35]. The described forces on the parallel flows also appear in the collisionless flow theory by Rosenbluth and Hinton [37]. The relevant construct is the odd sourceterm in equation (13) therein, which represents the difference between particle source rates with positive and negative momentum, in other words, the parallel momentum injection rate.

The importance of the parallel stress has been confirmed experimentally in the TJ-II stellarator by measurements with reciprocating Mach probe arrays [11]. The magnitude of the stress was such that by itself it would have changed the parallel Mach numbers on a time constant of the order of $100 \mu \mathrm{s}$. In agreement with the above mixing length arguments this corresponds to the relevant local energy transport time scales.

\section{Transition to geodesic acoustic modes}

Since in a tokamak edge (with $q>2-3$ ) the parallel flow component contains far more energy than the poloidal $\boldsymbol{E} \times \boldsymbol{B}$-flow, the losses due to the positive parallel viscosity outweigh the gains due to negative poloidal viscosity effects, ruling out stationary edge ZFs. GAMs are of course allowed, even facilitated by the elimination of the parallel flows, which would otherwise tend to short-circuit the associated pressure perturbations.

Core turbulence simulations have always yielded a peak at the GAM frequency [33], which, however, has a very weak effect on the turbulence. This is because the GAM frequency is much higher than the local turbulence frequencies, due to low gradients and high speed of sound (compare (6)). The turbulence averages over complete GAM periods, and the resulting net shearing effect on the turbulence is small.

In contrast, at the edge of a tokamak, the sound velocity is much smaller due to lower temperature, and the turbulence frequencies higher due to high gradients, whence the GAMs are as effective at shearing eddies as stationary flows. In addition, the wave trapping effects mentioned in section 4 in conjunction with the oscillation produce a characteristic modulation of the turbulence in time and space [32].

The modulated transport is the root of two different drive mechanisms specific to the GAMs (apart from the perpendicular Reynolds stress). On one hand, the shear flows break the up-down symmetry of the turbulence, causing an up-down asymmetric transport component. The transport asymmetry subsequently affects the pressure asymmetry [32] - one of the phases of the GAM -, amplifying or weakening 
it, depending on the relative phase of the transport asymmetry. The corresponding GAM growth rate has been computed for a simple turbulence asymmetry model in [48].

On the other hand, the oscillating transport modulates the pressure profile, whose gradients are proportional to the background diamagnetic velocity, $v_{\text {diamagnetic }}=$ $(|\nabla \ln n|+|\nabla \ln T|) T / B$. Neoclassical effects connect the diamagnetic velocity with the zero-point of the GAM oscillation. Consequently, the induced oscillation of this zero-point can transfer energy into the GAMs - just as moving the suspension excites a pendulum.

All these mechanisms can also extract energy from the GAMs, depending on the relative phase of the coupling term to the oscillation. In addition, the pressure fluctuations associated with the GAM are naturally subject to erosion due to anomalous or neoclassical transport effects. As will be discussed in more detail in the next section, toroidal effects couple the GAMs to parallel flows (or more accurately sound waves), at least to some degree. As with the stationary flows, these parallel degrees of freedom are subject to turbulent braking and collisional damping and thus represent another energy sink for the GAMs. Due to the finite frequency of the GAMs the parallel degrees of freedom are also Landau damped in the absence of dissipation, which leads to damping rates on the order of $\sim \omega_{\mathrm{G} A M} \exp \left(-q^{2}\right)$ [38].

\section{GAM frequency for real tokamaks}

Real tokamaks have of course shaped flux surface which are not concentric. Neglecting at first parallel flows, the effects of flux surface geometry may be described by two parameters. Because the flow potential is a flux surface quantity, the poloidal variation of the distance between flux surfaces causes an electric field variation and thus a variation of the $\boldsymbol{E} \times \boldsymbol{B}$-velocity. This modifies the flux surface averages entering the energy balance relation for the frequency (6). The averages, relative to the reference velocity $v_{\mathrm{p}, 0}$ at the outboard mid-plane, can be described by two geometrical coefficients for the kinetic and compressional energy,

$$
\begin{aligned}
& \left\langle v_{\mathrm{p}}^{2}\right\rangle=C_{1} v_{\mathrm{p}, 0}^{2}, \quad C_{1} \equiv \frac{\left\langle(|\nabla \psi| / B)^{2}\right\rangle}{\left|(\nabla \psi)_{0}\right|^{2} / B_{0}^{2}} \approx \frac{\left\langle(\nabla \psi)^{2}\right\rangle}{\left|(\nabla \psi)_{0}\right|^{2}}, \\
& \left\langle(\boldsymbol{v} \cdot \nabla \ln B)^{2}\right\rangle=C_{2} v_{\mathrm{p}, 0}^{2}, \quad C_{2}=\frac{\left\langle\left(F \partial_{\|} B^{-1}\right)^{2}\right\rangle}{\left|(\nabla \psi)_{0}\right|^{2} / B_{0}^{2}} \approx \frac{\left\langle\left(\partial_{z} \psi\right)^{2}\right\rangle}{\left|(\nabla \psi)_{0}\right|^{2} R_{0}^{2}},
\end{aligned}
$$

where $F \equiv B_{\phi} R$ is one of the flux surface quantities of the Grad-Shafranov equation and the index 0 signifies quantities taken at the reference position at the outboard midplane, $z$ is the vertical coordinate, and the approximation holds for $R \gg a$. Since $\psi$ is the flux-surface label, $(\nabla \psi)^{2}$ can be interpreted as the inverse squared distance and $\left(\partial_{z} \psi\right)^{2}$ as the inverse squared vertical distance of neighbouring flux surfaces as a function of $\theta$.

Applying the energy argument of section 3 one arrives at the frequency

$$
\omega=2 \sqrt{\gamma} c_{s} \sqrt{\frac{C_{2}}{C_{1}}}=2 \sqrt{\gamma} c_{s} \sqrt{\frac{\left\langle\left(F \partial_{\|} B^{-1}\right)^{2}\right\rangle}{\left\langle(|\nabla \psi| / B)^{2}\right\rangle}} \approx \frac{2 \sqrt{\gamma} c_{s}}{R_{0}} \sqrt{\frac{\left\langle\left(\partial_{z} \psi\right)^{2}\right\rangle}{\left\langle\nabla \psi^{2}\right\rangle}} .
$$




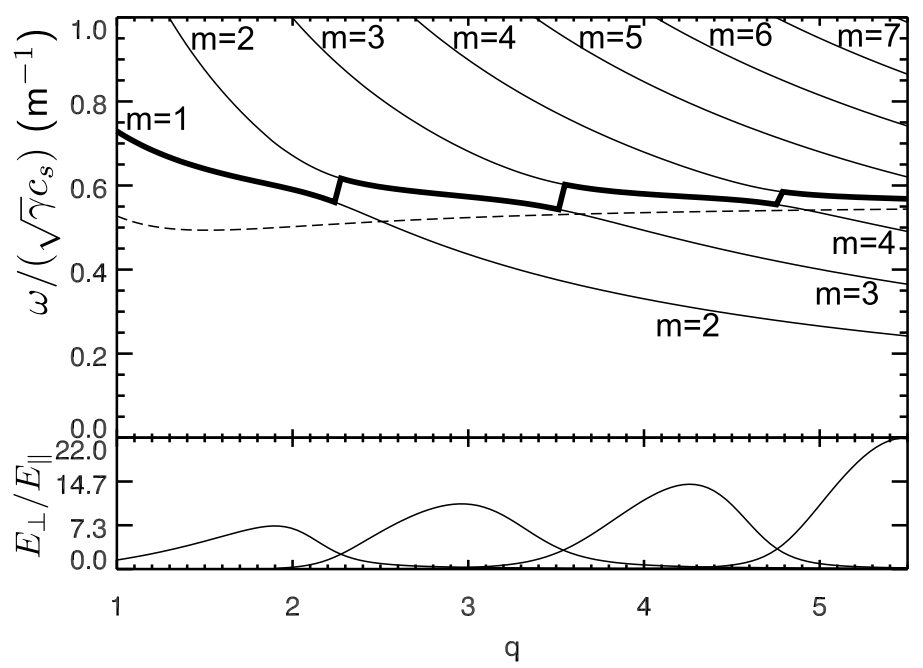

Figure 2. Top: Frequency of sound wave branches (thin solid), the GAM (thick), and pure GAM frequency (dashed) versus safety factor $q$. The dominant mode number $m$ of the parallel displacement field is indicated for each branch. Crossing of the GAM branch changes $m$ by one for each sound wave. Bottom: ratio of perpendicular to parallel kinetic energy; the sound wave branch with maximum ratio is the GAM. Equilibrium parameters: $R=1.6 \mathrm{~m}, a=0.5 \mathrm{~m}, \kappa=1.5$, $R^{\prime}=-0.3, a \kappa^{\prime} / \kappa=0.4$. The pure GAM frequency in a concentric circular plasma would be $\omega /\left(\sqrt{\gamma} c_{s}\right)=\sqrt{2} /(1.6 \mathrm{~m})=0.9 \mathrm{~m}^{-1}$.

For an elliptic Miller-equilibrium [49] in the high aspect ratio limit, the required ratio can be given analytically as

$$
\frac{\left\langle\left(\partial_{z} \psi\right)^{2}\right\rangle}{\left\langle\nabla \psi^{2}\right\rangle}=\frac{P}{1+\left(\kappa^{2}-1\right)(1-P)}, \quad P \equiv \frac{2}{\left(1+Q^{-1}\right)\left(1+\sqrt{1+\frac{2 a \kappa^{\prime} / \kappa}{1+Q}}\right)},
$$

where $Q \equiv \sqrt{1-R^{\prime 2}}, a$ is the minor radius at the midplane, $\kappa$ is the ellipticity, $R^{\prime}$ and $\kappa^{\prime}$ are the differential Shafranov shift and ellipticity. (The prime indicates derivative with respect to the minor radius at the midplane.)

Although the GAM frequency is normally not resonant with the parallel sound transit frequencies, the plasma will move a little along the field lines in response to the parallel pressure gradients caused by the GAM. The coupling turns the frequency calculation into an eigenvalue problem which can only be solved numerically [50].

Figure 2 shows an example spectrum of axisymmetric acoustic eigenmodes obtained numerically for a Miller-equilibrium [49], together with a plot of the "pure" GAM-frequency calculated without the coupling (note that the dependence on $q$ enters predominantly due to the finite aspect ratio in this case, and is absent for $R \gg a$ ). Since the pure GAM frequency has intersections with all the sound wave branches to which it couples, it is not trivial to identify the branch corresponding to the GAM in the absence of coupling. A little bit away from the resonances with individual acoustic branches, this can be done by selecting the mode with the highest ratio of perpendicular to parallel flow energy (bottom of figure 2 and top highlighted graph).

It is interesting to note, that the GAM switches between continuous acoustic branches at the resonances with the sound waves, and that the dominant mode 


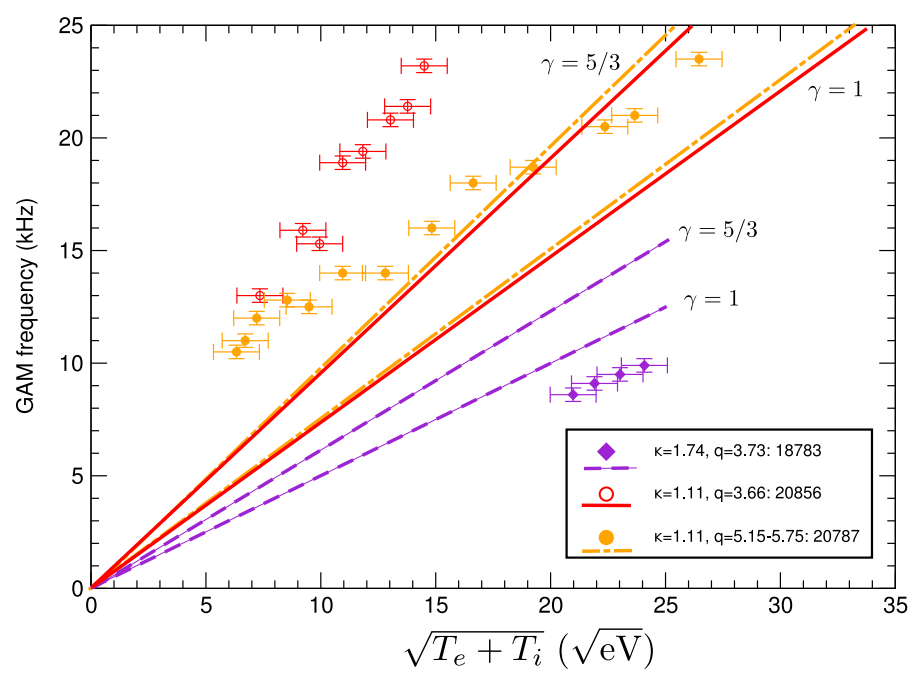

Figure 3. Measured GAM frequencies (points), and calculated linear GAM frequencies including sound wave coupling, using a Miller equilibrium [49] reproducing the parameters $R, r, R^{\prime}, \kappa, \kappa^{\prime}$ of respective the nominal equilibrium. Experimental data from [52].

number of a continuous branch is reduced by one, when $q$ moves from above the upper resonance to below the lower resonance.

These features can be understood by considering how the branches for the coupled spectrum arise from the pure, uncoupled modes. Firstly, the uncoupled pure GAM branch intersects with various sound wave branches as $q$ traverses from high to low values. The coupling between the GAM and the sound waves will tend to push the GAM frequency and the closest sound frequency branch away from each other. Each branch-crossing is resolved into two smooth transitions between the GAM branch and one of the sound wave branches. As result, the GAM-branch is broken down into several segments, which connect sound-wave branches with poloidal mode number differing by one.

Particularly strong damping due to the parallel turbulent stresses (see previous sections) is expected at the resonances with the sound waves, i.e., at the branch switching locations. This could be an explanation for the experimentally observed windows of GAM activity in D3D [51].

Let us now compare the linear mode frequencies with experimental values from ASDEX Upgrade [52] (figure 3). Since the strength of kinetic effects and parallel heat conduction may be debated, the linear mode frequencies have been plotted for the range of adiabatic coefficients from $\gamma=5 / 3$ (no heat conduction) to $\gamma=1$ (infinite parallel heat conduction, isothermal), where the nominal value is $\gamma=4 / 3$ (see section 3). Although the frequencies generally follow the scaling with the sound velocity $c_{s} \propto \sqrt{T}$, good agreement occurs only for the data points from the core plasma inside the pedestal (discharge 20787 for $\sqrt{T_{e}+T_{i}}>20 \sqrt{\mathrm{eV}}$ ). In the high gradient regions of the edge (outside the pedestal) the experimental frequencies are either higher (discharges 20856, and 20787 for $\sqrt{T_{e}+T_{i}}<20 \sqrt{\mathrm{eV}}$ ) or lower (discharge 18783) than the calculated linear frequencies. 

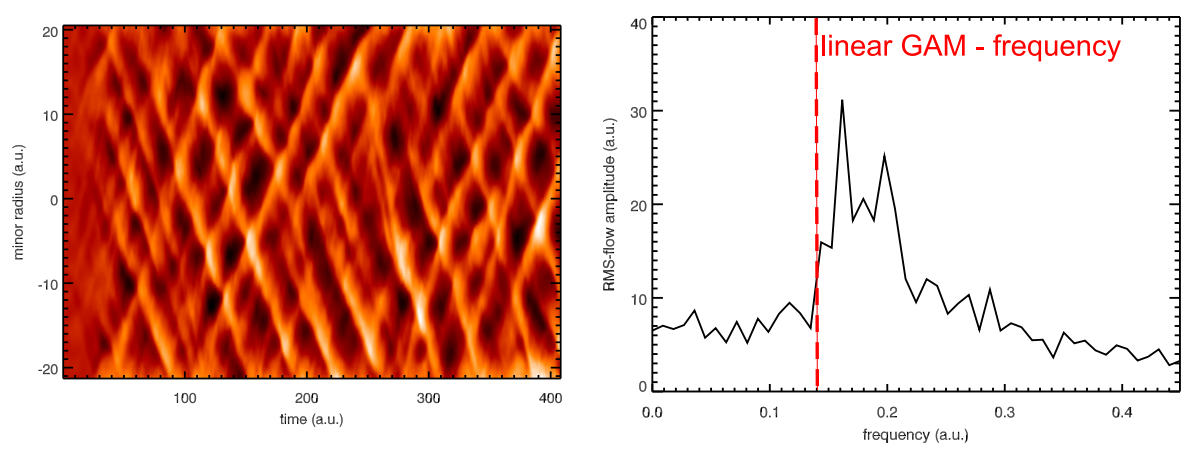

Figure 4. Left: Colour coded flow velocity $\left\langle v_{\theta}\right\rangle$ versus minor radius and time. Right: Linear GAM-frequency and Fourier transformed flow spectrum, which is nonlinearly upshifted by $30 \%$. Simulation parameters (for definition see [32] and references therein) $\alpha_{d}=1, \epsilon_{n}=0.03, \tau=1, q=3.2, s=1, \eta_{i}=4$.

The high gradient regions of the edge plasma are notoriously hard to characterise, and the deviation could be simply due to slight inaccuracies of the magnetic structure there. Another possibility is the turbulence itself: The turbulence-flow interaction terms could just as well push the GAM instead of driving it, i.e., change the real GAMfrequency instead of just providing the imaginary part corresponding to the growth of the flows. Indeed, in turbulence simulations at comparatively high gradients the GAM frequency can be up to about $30 \%$ larger than the linear value (figure 4).

\section{Summary}

Analogous to the well known zonal winds in the atmosphere of gas planets, the plasma zonal flows are excited due to the quasi-2D-restriction of the turbulence perpendicular to the magnetic field or the axis of rotation, respectively. Different from the purely 2D planetary zonal winds, the inhomogeneous magnetic fields frozen into the moving plasma excite strong flows parallel to the field, as the circulating flux ropes compress or expand, to adjust to the ambient conditions. Depending on the magnetic geometry, the parallel flow may have the effect of a changed effective inertia (typically in tokamak core, where the safety factor is around one) or can be such a strong energy drain that a stationary flow pattern becomes impossible and an oscillation between plasma compression and flow results (usually in the tokamak edge, with $q \sim 3-5$ ).

This 3D-nature of the plasma flows causes a complex interplay of the parallel turbulent stresses, cross field transport, and coupling to parallel sound waves, which is absent in "flat" slab or cylindrical models. As for fusion applications, these notions have predicted testable connections between parallel flows and Reynolds stress on one hand, and the perpendicular flow on the other. They can explain the recently observed radial windows of GAM activity and open new possibilities for transport reduction by designing optimal geometries for the flows.

More general, the described effects are expected for any type of curved flux surface, which opens a so far untapped field of applications in solar and astrophysical plasmas, an example of which are the oscillations of coronal loops.

[1] McKee G R et al 2003 Phys. Plasmas 51712

[2] Winsor N et al 1968 Phys. Rev. Lett. 112448

[3] Conway G D et al Plasma Phys. Control. Fusion 47, 1165 (2005) 
[4] Fujisawa A et al 2004 Phys. Rev. Lett. 93165002

[5] Shats M G et al 2006 Plasma Phys. Control. Fusion 48 S17

[6] Zhao K J et al 2006 Phys. Rev. Lett. 96255004

[7] Ido T et al 2006 Nucl. Fusion46 512

[8] Marchenko V S et al 2006 Phys. Plasmas 13060701

[9] Hamada Y et al 2005 Nucl. Fusion45 81

[10] Krämer-Flecken A et al 2006 Phys. Rev. Lett. 97045006

[11] Goncalves B et al 2006 Phys. Rev. Lett. 96145001

[12] Melnikov A V et al 2006 Plasma Phys. Control. Fusion 48 S87

[13] Hasegawa A et al 1979 Phys. Fluids 222122

[14] Diamond P H, Kim Y-B 1991 Phys. Fluids B 31626

[15] Hasegawa A et al 1987 Phys. Rev. Lett. 591581

[16] Hammett G W et al 1993 Plasma Phys. Control. Fusion 35973

[17] Lin Z et al 1998 Science $\mathbf{2 8 1} 1835$

[18] Champeaux S et al 2001 Phys. Lett. A 288214

[19] Hallatschek K et al 2003 New J. Phys. 529.1

[20] Stringer T E 1969 Phys. Rev. Lett. 22770

[21] Itoh K et al 2005 Plasma Phys. Control. Fusion 47451

[22] Chapman C R 1969 J. Atmos. Sci. 26986

[23] Busse F H 1994 Chaos 4123

[24] Marcus P S et al 1994 Chaos 4269

[25] Hallatschek K 2004 Phys. Rev. Lett. 931250001

[26] McCarthy D R 1993 Phys. Fluids B 52145

[27] Parker S E et al 1999 Phys. Plasmas 61709

[28] Dimits A M et al 2000 Phys. Plasmas 7969

[29] Biglari H et al 1990 Phys. Fluids B 21

[30] Mattor N 1994 Phys. Plasmas 2766

[31] Connor J W et al 2000 Plasma Phys. Control. Fusion 42 R1

[32] Hallatschek K et al 2001 Phys. Rev. Lett. 861223

[33] Hahm T S et al 2000 Plasma Phys. Control. Fusion 42 A205

[34] Chen L et al 2000 Phys. Plasmas 73129

[35] Hallatschek K 2004 Phys. Rev. Lett. 9365001

[36] Miyato N et al 2004 Phys. Plasmas 115557

[37] Rosenbluth M N et al 1997 Phys. Rev. Lett. 80724

[38] Hinton F L et al 1999 Plasma Phys. Control. Fusion 41 A653

[39] Sugama H et al 2005 Phys. Rev. Lett. 94115001

[40] Watari T et al 2005 Phys. Plasmas 12062304

[41] Hinton F L, Rosenbluth M N and Diamond P H, Radial Propagation of GAMs and Zonal Flows. In International Sherwood Fusion Theory Meeting, Santa Fe, 2001.

[42] Wang T et al 2002 ApJ $\mathbf{5 7 4}$ L101

[43] Kraichnan R H et al 1980 Rep. Prog. Phys. 43547

[44] Itoh K et al 2005 Phys. Plasmas 12062303

[45] Lebedev V B et al 1995 Phys. Plasmas 24420

[46] Montgomery D et al Plasma Phys. Control. Fusion 26717

[47] Nishijima D et al 2005 Plasma Phys. Control. Fusion 4789

[48] Itoh K et al 2005 Plasma Phys. Control. Fusion 47451

[49] Miller R L et al 1998 Phys. Plasmas 5973

[50] Nührenberg C et al 2006 33rd EPS Conf. on Control. Fusion and Plasma Physics (Rome, Italy) paper P2.116

[51] McKee G R et al 2006 Plasma Phys. Control. Fusion 48 S123

[52] Conway G D et al 2007 34th EPS Conf. on Control. Fusion and Plasma Physics (Warsaw, Poland) paper O4.009; Conway et al "Geodesic acoustic mode scaling and core localization in ASDEX Upgrade using Doppler reflectometry" Plasma Phys. Control. Fusion to be published 